Combined selective emitter and filter for high performance incandescent lighting

Arny Leroy, Bikram Bhatia, Kyle Wilke, Ognjen Ilic, Marin Soljačić, and Evelyn N. Wang

Citation: Appl. Phys. Lett. 111, 094103 (2017); doi: 10.1063/1.4989522

View online: http://dx.doi.org/10.1063/1.4989522

View Table of Contents: http://aip.scitation.org/toc/apl/111/9

Published by the American Institute of Physics

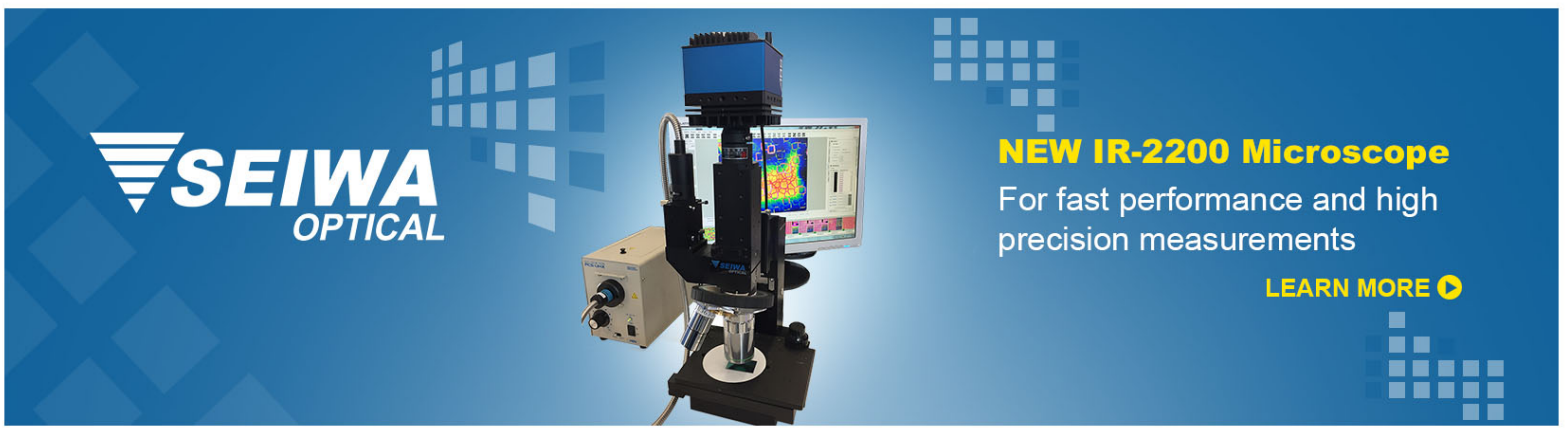




\title{
Combined selective emitter and filter for high performance incandescent lighting
}

\author{
Arny Leroy, ${ }^{1}$ Bikram Bhatia, ${ }^{1}$ Kyle Wilke, ${ }^{1}$ Ognjen Ilic, ${ }^{2}$ Marin Soljačić,, 4 \\ and Evelyn N. Wang ${ }^{1}$ \\ ${ }^{1}$ Device Research Laboratory, Department of Mechanical Engineering, Massachusetts Institute of Technology, \\ 77 Massachusetts Avenue, Cambridge, Massachusetts 02139, USA \\ ${ }^{2}$ Department of Applied Physics and Materials Science, California Institute of Technology, \\ 1200 East California Boulevard, Pasadena, California 91125, USA \\ ${ }^{3}$ Research Laboratory of Electronics, Massachusetts Institute of Technology, 77 Massachusetts Avenue, \\ Cambridge, Massachusetts 02139, USA \\ ${ }^{4}$ Institute for Soldier Nanotechnology, Massachusetts Institute of Technology, 77 Massachusetts Avenue, \\ Cambridge, Massachusetts 02139, USA
}

(Received 9 June 2017; accepted 14 August 2017; published online 1 September 2017)

\begin{abstract}
The efficiency of incandescent light bulbs (ILBs) is inherently low due to the dominant emission at infrared wavelengths, diminishing its popularity today. ILBs with cold-side filters that transmit visible light but reflect infrared radiation back to the filament can surpass the efficiency of state-ofthe-art light-emitting diodes (LEDs). However, practical challenges such as imperfect geometrical alignment (view factor) between the filament and cold-side filters can limit the maximum achievable efficiency and make the use of cold-side filters ineffective. In this work, we show that by combining a cold-side optical filter with a selective emitter, the effect of the imperfect view factor between the filament and filter on the system efficiency can be minimized. We experimentally and theoretically demonstrate energy savings of up to $67 \%$ compared to a bare tungsten emitter at $2000 \mathrm{~K}$, representing a 34\% improvement over a bare tungsten filament with a filter. Our work suggests that this approach can be competitive with LEDs in both luminous efficiency and color rendering index (CRI) when using selective emitters and filters already demonstrated in the literature, thus paving the way for next-generation high-efficiency ILBs. Published by AIP Publishing.

[http://dx.doi.org/10.1063/1.4989522]
\end{abstract}

The residential and commercial sectors in the United States used approximately $279 \times 10^{9} \mathrm{kWh}^{1}$ of electricity for lighting in 2016 , accounting for $10 \%$ of the total electricity consumption of these sectors. Incandescent light bulbs (ILBs), which are still widely installed, are typically characterized by a perfect and more desirable color rendering index ${ }^{2}$ (CRI; capacity to faithfully reproduce colors of the illuminated object) of 100 but with a relatively low luminous efficiency $^{3,4}$ (comparison of luminous flux to power consumption; detailed definition available in the supplementary material) of $1.5 \%-3 \%$ (equivalent to the luminous efficacy of $10-22 \mathrm{~lm} / \mathrm{W})$. Meanwhile, typical commercial light-emitting diode (LED) bulbs have higher luminous efficacies of 61-140 lm/W, ${ }^{4}$ with world-record LED efficacies approaching $303 \mathrm{~lm} / \mathrm{W},{ }^{5}$ but often have a lower CRI in the 70 s to $90 \mathrm{~s}$ which is less desirable. By steadily adopting more efficient light sources with efficiencies comparable to LEDs, it is projected that by 2035 , a $75 \%$ energy consumption reduction in lighting can be achieved, thus providing cumulative energy savings of nearly $\$ 630$ billion. $^{6}$

ILBs operate by heating a tungsten filament at incandescent temperatures in an inert environment. While the temperature of the filament can be increased to have a greater portion of the blackbody spectrum within the visible spectrum and thus higher luminous efficiency, its temperature is in practice limited to $\sim 3000 \mathrm{~K}$ due to filament evaporation which affects the lifetime and darkens the bulb. The efficiency of ILBs can also be improved by spectrally tailoring the emitted radiation using a cold-side interference filter [Fig. 1(a)] which minimizes the heat losses due to undesired infrared emission. This approach was first proposed in $1912^{7}$ and has since been extensively investigated. ${ }^{8-30}$ Several studies attempted to maximize the light source efficiency while maintaining a high CRI by exploring different types of filters (silver films with $\mathrm{TiO}_{2}$ antireflection coatings, ${ }^{7,10,11,28,29}$ $\mathrm{TiO}_{2}-\mathrm{SiO}_{2}{ }^{15}$ and $\mathrm{Ta}_{2} \mathrm{O}_{5}-\mathrm{SiO}_{2}{ }^{8,17-19,21,22,30}$ multilayer films, doped semiconductors $\left(\mathrm{In}_{2} \mathrm{O}_{3}: \mathrm{Sn}\right),{ }^{16}$ and silver photonic crystals $\left.{ }^{23}\right) . \mathrm{Ta}_{2} \mathrm{O}_{5}-\mathrm{SiO}_{2}$ multilayer films appeared to offer the best compromise between cost, optical properties, and thermal stability (up to $800^{\circ} \mathrm{C}^{19,22,30}$ ). Concurrently, several bulb geometries (spherical, $^{10,11,16,28,29,31,32}$ cylindrical, ${ }^{7,8,14,15,17,19,23,30}$ ellipsoidal, ${ }^{18,22,29}$ and planar $^{21}$ ) were studied to reduce fabrication complexity and maximize the amount of recycled infrared radiation while minimizing hot spots on the filament which can reduce its lifetime. The emitter in all these past studies using selective filters was typically a tungsten filament, chosen due to its high temperature stability and low evaporation rate at incandescent temperatures. Although extensive research has been performed, only limited energy savings were demonstrated (up to $51 \%$ lower energy consumption compared to typical ILBs, ${ }^{10}$ corresponding to an estimated $4 \%-5 \%$ luminous efficiency) due to non-idealities in the coldside filters (low infrared reflectivity and/or low visible transmissivity), non-idealities in the tungsten filaments (relatively low visible and high infrared emissivity), challenges in the deposition of interference films on curved bulbs, high filter 
(a)

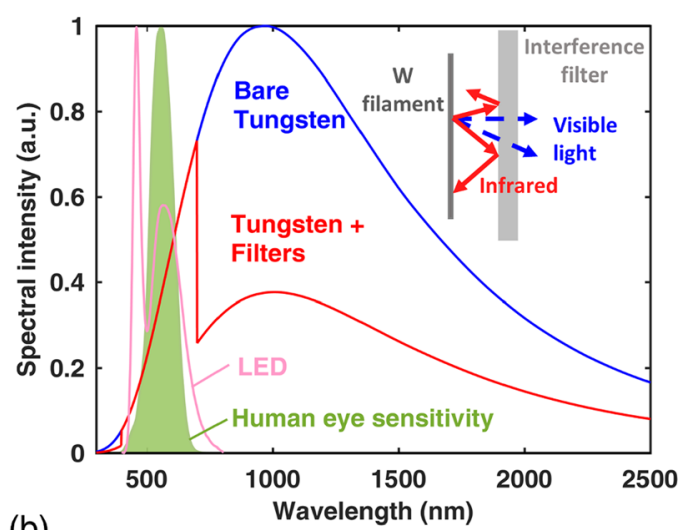

(b)

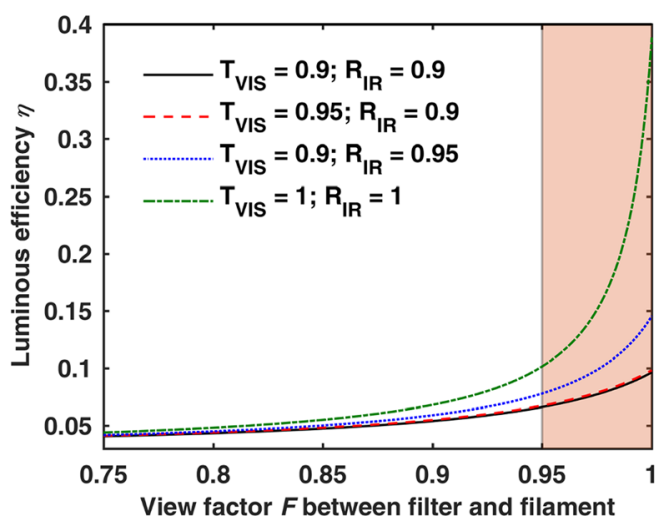

FIG. 1. (a) Spectral intensity for a bare planar tungsten emitter and for a tungsten emitter surrounded with planar selective filters (view factor of $F=0.95$; for the filters, $R=1-T$ is assumed, with typical high performance infrared reflectivity $R_{I R}=0.9$ and visible transmissivity $T_{V I S}=0.95$ ). Spectral tailoring using selective filters allows a decrease in the infrared emission and an increase in the luminous efficiency of the light source. The photopic human eye sensitivity curve and the spectrum of a typical white $\mathrm{LED}^{33}$ are shown for reference. See supplementary material for details of modeling and temperature dependent spectral emissivity of tungsten (Fig. S3). (b) Influence of the filament-filter view factor on the luminous efficiency of a system with a tungsten filament at $2800 \mathrm{~K}$ and surrounding filters with different optical properties. Higher view factor, infrared reflectivity, and visible transmissivity lead to higher luminous efficiencies $(\eta)$, with a maximum $\eta=10.1 \%$ at $R_{I R}=1$ and $T_{V I S}=1$ for $F \leq 0.95$.

operating temperatures, and perhaps most importantly, imperfect geometrical alignment or view factor of the filament with the infrared mirror. The view factor $F$ between the filament and the filter represents the fraction between the radiation reaching the filter and the total diffuse radiation emitted from the filament, where non-idealities in this view factor characterize the fraction of the emitted radiation leaving the system without interacting with the filters. High view factors maximize the infrared radiation reflected by the filters back to the filament and are thus necessary to achieve high efficiencies.

The imperfect view factor $F$, typically $\leq 0.95$ for ILBs, fundamentally limits the efficiency of the system by reducing the recycling of infrared light by the filter and increasing the effective infrared emission of the system. This effect of the non-ideal view factor on the ILB luminous efficiency is shown in Fig. 1(b) for a planar filament-filter system consisting of a filament at $2800 \mathrm{~K}$ and surrounding filters with different visible and infrared optical properties. As expected, the luminous efficiency increases with the view factor as well as the filter infrared reflectivity $R_{I R}$ and visible transmissivity $T_{V I S}$. Figure 1 (b) also shows that $R_{I R}$ of the filter has a bigger influence on the luminous efficiency than $T_{V I S}$ in the range of the optical properties considered because of the dominant emission of infrared radiation by the filament (see supplementary material, Fig. S2). However, it also shows that most improvements in efficiency occur for $F>0.9$ and that when $F \leq 0.95$, the maximum efficiency (achieved at $R_{I R}=1$ and $T_{v i s}=1$ ) remains relatively low at just over $10 \%$, far from the theoretical value of $39.6 \%$ for a blackbody at $2800 \mathrm{~K}$ truncated to the visible range $(400-700 \mathrm{~nm})$ only. Figure 1(b) therefore suggests that system-level non-idealities such as imperfect view factor typically observed in real systems greatly impede the maximum efficiency and that improvements can still be made to reach higher and more competitive luminous efficiencies.

We propose an approach that combines a cold-side filter with a selective emitter instead of a typical tungsten filament to reduce the relative emission of infrared radiation. The potential of this approach is shown in Fig. 2 where the luminous efficiency of an ILB with $F=0.95$ is plotted as a function of the effective visible $\left(\varepsilon_{V I S}\right)$ and infrared $\left(\varepsilon_{I R}\right)$ emissivity of the emitter for fixed filter properties $\left(R_{I R}=0.9\right.$ and $\left.T_{V I S}=1\right)$. As expected, reducing the emitter's emissivity at infrared wavelengths and maximizing its emissivity at visible wavelengths increase the luminous efficiency, with most important gains in efficiency achieved at low infrared emissivity. In addition, Fig. 2 shows that higher efficiencies (up to $39.6 \%$ ) can be achieved at $F=0.95$ using a non-ideal selective emitter combined with a non-ideal filter as compared to using a tungsten filament with an ideal filter [up to 10.1\%; Fig. 1(b)]. Using a selective emitter therefore reduces the importance of non-idealities of the filters and view factor and allows for high luminous efficiency and CRI incandescent lighting that can be competitive with currently available LEDs.

We experimentally demonstrate the potential of this approach by comparing the emission spectra and power consumption of two different planar incandescent emitters (tungsten and selective emitter) with and without planar optical filters ${ }^{21}$ [see Fig. S4 (supplementary material) for filter

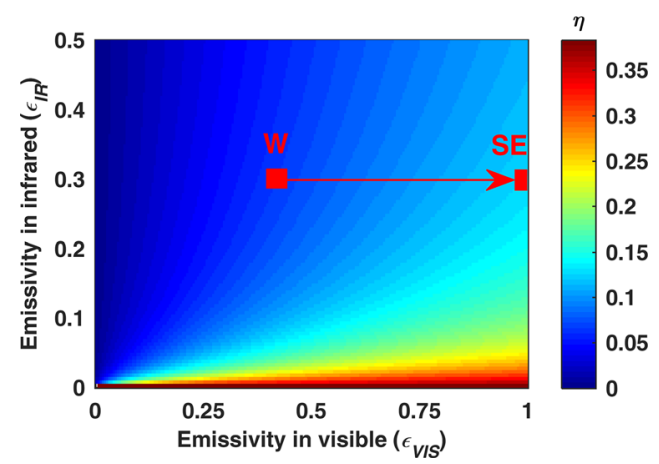

FIG. 2. Influence of a selective emitter's emissivity in the visible and infrared regions on the luminous efficiency of the system when combined with an optical filter of $R_{I R}=0.9$ and $T_{V I S}=1$, at a temperature of $2800 \mathrm{~K}$ and $F=0.95$. A higher emissivity in the visible and lower emissivity in the infrared lead to higher luminous efficiency. Increasing the emissivity in the visible region of a tungsten filament from $\varepsilon_{V I S}=0.42$ (tungsten emitter, W) to $\varepsilon_{V I S}=1$ (selective emitter, SE) while keeping the infrared emissivity constant increases the luminous efficiency from 6.5 to $12.5 \%$. 
(a)

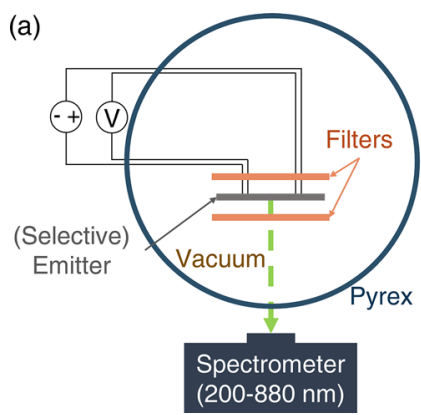

(b)

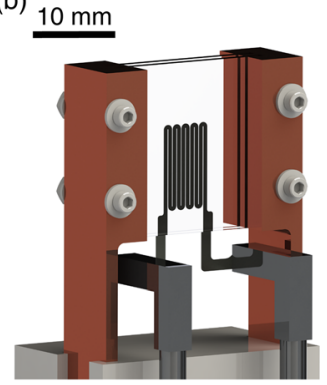

FIG. 3. (a) Schematic (top view) and (b) computer-aided design (CAD) rendering of the experimental setup. A planar radiator-like tungsten filament, fastened to electrical feedthroughs, is sandwiched between planar optical filters (transparent) which are held by copper supports for efficient heat dissipation.

optical properties] in a vacuum chamber (Fig. 3). As a proof of concept, a relatively simple selective emitter is fabricated by coating a thin $(55 \mathrm{~nm})$ antireflection layer of $\mathrm{HfO}_{2}$ by atomic layer deposition (see supplementary material) on a planar radiator-like tungsten emitter [Fig. 3(b)] which increases its effective emissivity in the visible spectrum [Fig. 4(a)]. The radiator-like geometry of the filament maximizes the planar surface area for increased reabsorption of infrared radiation while allowing for resistive heating. $\mathrm{HfO}_{2}$ was chosen for its low vapor pressure and high temperature

stability, ${ }^{34-36}$ and the film thickness was optimized to maximize luminous efficiency (Fig. S5 of the supplementary material). Figure 4(a) shows the room temperature emissivity of tungsten $(W)$ and $\mathrm{HfO}_{2}$ coated tungsten (Coated $W$ ) from theoretical simulations (see supplementary material) as well as measurements on the UV-Visible spectrophotometer (Carry-6000i), which are in good agreement. As desired, a significant increase in the visible emissivity for the $\mathrm{HfO}_{2}$ coated filament is observed.

We performed a high temperature demonstration of the spectral enhancement due to the $\mathrm{HfO}_{2}$ coating by comparing the emission spectrum $(400-887 \mathrm{~nm})$ in a range of temperatures $(350-2240 \mathrm{~K})$ of a bare planar tungsten filament (taken as a reference) with one of a $\mathrm{HfO}_{2}$ coated filament, both with and without optical filters. The filaments were resistively heated in a vacuum chamber (Fig. 3) at a pressure below $10^{-6}$ Torr, and their resistance, used to estimate the temperature of the filament (see supplementary material), was measured using a four-wire measurement technique. A spectrometer (USB4000 Ocean Optics), located outside the vacuum chamber, measured the emitted spectrum in the range of $400-887 \mathrm{~nm}$ in increments of $0.2 \mathrm{~nm}$ at the normal incidence angle. By comparing the measured emission spectra of a tungsten filament and a $\mathrm{HfO}_{2}$ coated filament at the same temperature, we calculated the spectral intensity ratio which (a)

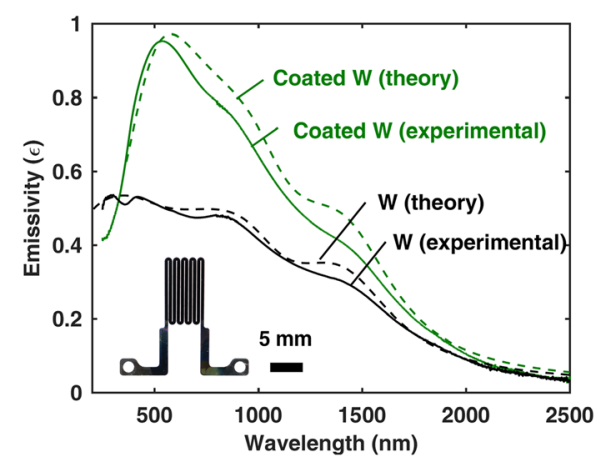

(c)

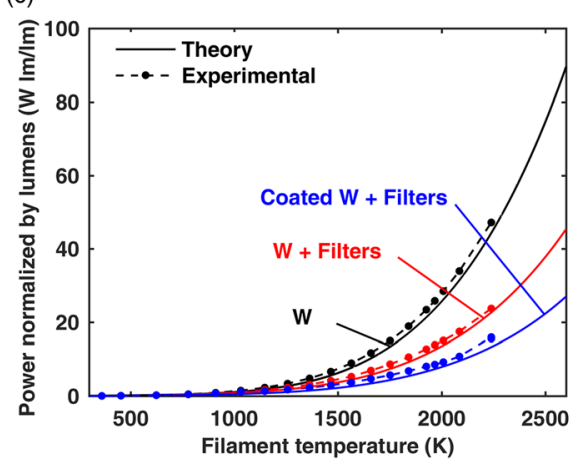

(b)

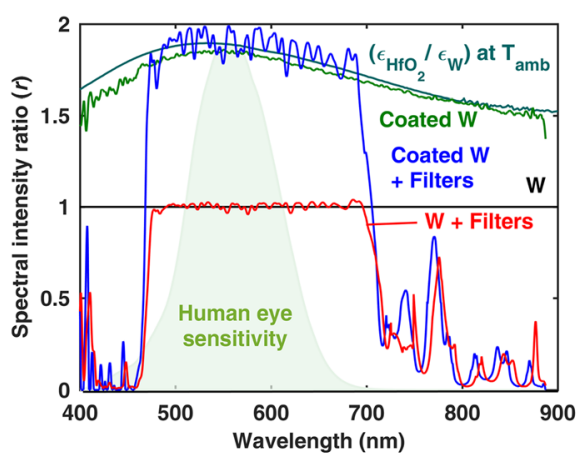

(d)

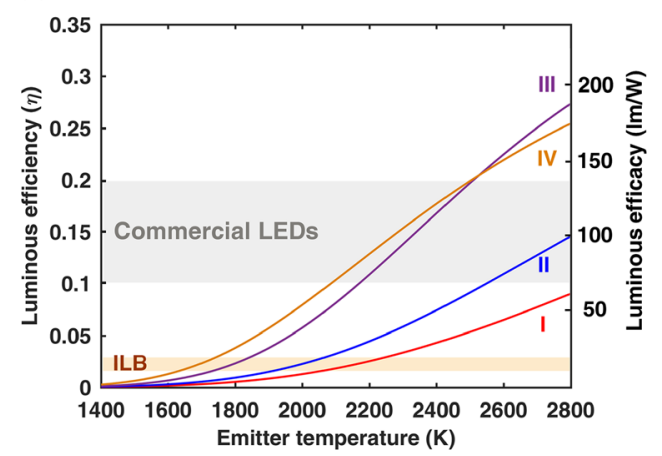

FIG. 4. (a) Theoretical and experimental room temperature emissivity of tungsten and $\mathrm{HfO}_{2}$ coated tungsten. The $\mathrm{HfO}_{2}$ coated tungsten filament is shown in the inset. (b) Experimental demonstration of the spectral enhancement in the range of $400-887 \mathrm{~nm}$ when using a selective emitter ( $\mathrm{HfO}_{2}$ coated W) and optical filters as compared to a bare tungsten emitter (W). Filament temperatures at which spectra were taken ranged between 1800 and $2100 \mathrm{~K}$ for higher emission at short wavelengths. The maximum and average experimental error on the spectral intensity ratio in the range of $450-850 \mathrm{~nm}$ is 0.24 and 0.04 , respectively, based on the combined instrumental error and 95\% confidence interval on precision error. (c) Filament power consumption normalized by emitted lumens as a function of filament temperature. The view factor between the filament and the filter was approximately 0.93 . The experimental error on power was smaller than the symbols (maximum: $\pm 0.1 \mathrm{~W}$ ). (d) Luminous efficiency $(\%)$ and efficacy $(\mathrm{lm} / \mathrm{W})$ using different selective emitters and filters currently available in the literature [see Fig. S10 (supplementary material) for optical properties] for $F=0.99$ with corresponding CRI at $2400 \mathrm{~K}$ : (I) Tungsten filament + current filter with $\mathrm{CRI}=91$; (II) $\mathrm{HfO}_{2}$ coated tungsten filament + current filter with $\mathrm{CRI}=89$; (III) Nanoimprinted superlattice metallic photonic crystal ${ }^{38}+600$-layer interference filter ${ }^{39}$ with CRI =90; (IV) $\mathrm{HfO}_{2}$ coated tantalum ${ }^{34}+2 \mathrm{D}$ metallic photonic crystal filter ${ }^{23}$ with CRI =94. Typical ILB and commercial LED luminous efficacies ${ }^{4}$ are shown in shaded areas for comparison and are independent of the x-axis (i.e., the efficacies are taken at the nominal operating conditions of the corresponding light sources which are not related to the "Emitter temperature (K)" plotted on the X-axis). 
represents the spectral ratio of the emissivity of the two filaments or the spectral increase in emission.

The results of the spectral intensity ratio for different configurations (with/without filters and with/without $\mathrm{HfO}_{2}$ coating) are plotted in Fig. 4(b). Good agreement is demonstrated between the spectral intensity ratio of $\mathrm{HfO}_{2}$ coated tungsten and tungsten only filaments as measured by the UV-Visible spectrophotometer at room temperature $\left(\left(\varepsilon_{H f O 2 / \varepsilon_{W}}\right)\right.$ at $\left.T_{a m b}\right)$ and the spectrometer (Coated $W$ ) at incandescent $(1800 \mathrm{~K}$ to $2100 \mathrm{~K})$ temperatures. For the $\mathrm{HfO}_{2}$ coated tungsten filament with no optical filters (Coated $W$ ), an average spectral enhancement ratio of 1.91 is observed in the visible spectrum with only a $5.2 \%$ increase in power consumption at $2090 \mathrm{~K}$. It is also demonstrated that this increase in visible emission due to the $\mathrm{HfO}_{2}$ is maintained when using optical filters (Coated $W+$ Filters) and that, as previously demonstrated in the literature, significant reduction in the near infrared emission due to the filters can be achieved.

To further illustrate the benefits of the proposed approach to combine the selective emitter and optical filter (Coated $W+$ Filters) compared to a plain tungsten filament with $(W+$ Filters) or without filters $(W)$, the filament power consumption normalized by the number of lumens (radiant emission weighed by the human eye sensitivity function) is plotted as a function of filament temperature in Fig. 4(c). Experimental energy savings of up to $50 \%$ are observed when adding filters to a tungsten filament $(W+$ Filters) while savings reach up to $67 \%$ when using a $\mathrm{HfO}_{2}$ coated tungsten filament with filters (Coated $W+$ Filters), thus providing a further $34 \%$ improvement by using a selective emitter as opposed to previous approaches using only a selective filter. Good agreement is also shown between theoretical and experimental curves of the normalized power consumption as a function of temperature. In the current system, a significant increase in the visible emissivity only slightly increases the power consumption because of the visible spectrum representing only a fraction of the blackbody spectrum at the temperatures tested, thus greatly improving the luminous efficiency. In addition to achieving increased energy savings using a selective emitter, a high CRI of 93 is calculated ${ }^{2,37}$ (at $2000 \mathrm{~K}$ ), giving the light source a competitive and desired quality of faithfully reproducing colors. The maximum temperature of the filament during the experiment was however limited by the degradation and evaporation of the $\mathrm{HfO}_{2}$ thin film (see supplementary material), leading to reduced visible emission, increased power consumption, and filter darkening over time.

While the experimental demonstration is only a proof of concept and many practical challenges such as temperature stability remain, the proposed approach suggests the potential to achieve high efficiency incandescent lighting. By using selective emitters and filters currently available in the literature ${ }^{21,23,34,38}$ with better spectral selectivity (Fig. S10 of the supplementary material), we demonstrate in Fig. 4(d) the potential of our proposed approach to combine the selective emitter and filter and show that high luminous efficiency and CRI incandescent lighting can be competitive with stateof-the-art commercial LEDs, even at significantly lower temperatures than current ILBs. Comparison with LEDs in Fig. 4(d) also allows for contextualization and better understanding of the required emitter and filter optical properties as well as emitter temperature for incandescent lighting to be competitive with LEDs. Lower filament temperatures in incandescent lighting can also have beneficial effects such as lower filter temperatures leading to higher thermal stability, longer filament lifetime, as well as smaller parasitic heat losses [e.g., conduction losses through electrical connections, and conduction and convection losses to noble gas; parasitic heat losses were not considered for Fig. 4(d)]. While high temperature stability remains the foremost challenge for selective emitters in incandescent lighting, the development of thermally stable selective emitters could pave the way for a new generation of highly efficient light sources with high CRI as well as be useful in thermophotovoltaic applications.

In summary, we propose an approach that combines a selective emitter with cold-side optical filters to simultaneously achieve high luminous efficiency and high CRI in ILBs. While previous approaches mainly focused on developing high performance cold-side filters, we show that the non-ideal view factor between the filament and cold-side filters observed in practice due to geometrical constraints significantly limits the maximum achievable luminous efficiency. By using a selective emitter with cold-side filters, we have theoretically and experimentally demonstrated improved energy savings of up to $67 \%$ compared to a bare tungsten emitter at $2200 \mathrm{~K}$. Finally, when using selective emitters and filters already demonstrated in the literature, our proposed approach shows the potential to be competitive in luminous efficiency with other lighting technologies such as LEDs while still possessing the superior CRI characteristic of ILBs.

See supplementary material for more information on select topics.

This work was primarily supported as part of the SolidState Solar Thermal Energy Conversion (S3TEC) Center, an Energy Frontier Research Center funded by the U.S. Department of Energy, Office of Science, Basic Energy Sciences under Award No. \#DE-FG02-09ER46577. A. Leroy acknowledges funding received from the Fonds de Recherche du Québec—Nature et Technologies (FRQNT).

${ }^{1}$ See https://www.eia.gov/tools/faqs/faq.php?id=99\&t=3 for "How much electricity is used for lighting in the United States? - FAQ - U.S. Energy Information Administration (EIA)" (last accessed March 24, 2017).

${ }^{2}$ G. Wyszecki and W. S. Stiles, Color Science, 2nd ed. (Wiley, New York, 1982).

${ }^{3}$ T. W. Murphy, J. Appl. Phys. 111, 104909 (2012).

${ }^{4}$ See https://energy.gov/eere/energybasics/articles/lighting-basics for "Lighting Basics I Department of Energy" (last accessed March 24, 2017).

${ }^{5}$ See http://www.cree.com/news-media/news/article/cree-first-to-break-

300-lumens-per-watt-barrier for "Cree First to Break 300 Lumens-PerWatt Barrier" (last accessed May 15, 2017).

${ }^{6}$ Navigant Consulting, Inc., "Energy Savings Forecast of Solid-State Lighting in General Illumination Applications," U.S. Dept. of Energy Technical Report DOE/EE-1467, September 2006.

${ }^{7}$ S. O. Hoffman, "Production of light," U. S. patent 1,043,008 (1912).

${ }^{8}$ R. S. Bergman, J. Illum. Eng. Soc. 20, 10 (1991).

${ }^{9}$ R. S. Bergman and T. G. Parham, Sci. Meas. Technol. IEE Proc. A 140, 418 (1993).

${ }^{10}$ L. Brett, J. Fontana, R. Walsh, P. Spura, S. Parascandola, L. Thouret, and W. Thorington, J. Illum. Eng. Soc. 10, 214 (1981). 
${ }^{11}$ J. Brett, R. P. Fontana, P. J. Walsh, and S. A. Spura, J. Illum. Eng. Soc. 9, 197 (1980).

${ }^{12}$ J. Brett, R. P. Fontana, P. J. Walsh, W. E. Thouret, M. Gerber, R. Kaufman, and L. Thorington, IEEE Trans. Ind. Appl. IA-17, 210 (1981).

${ }^{13}$ J. R. Coaton and J. R. Fitzpatrick, IEE Proc., Part A: Phys. Sci. Meas. Instrum., Manage. Educ. Rev. 127(3), 142 (1980).

${ }^{14}$ T. K. Hoegler and L. E. McGowan, J. Illum. Eng. Soc. 14, 165 (1984).

${ }^{15}$ K. Honda, A. Ishizaki, Y. Yuge, and T. Saitoh, Proc. SPIE 428, 29 (1983).

${ }^{16}$ H. Kostlin and G. Frank, Philips Tech. Rev. 41, 225 (1983).

${ }^{17}$ R. L. Martin, Jr., Opt. News 12, 23 (1986).

${ }^{18}$ Y. Omata, N. Hashimoto, S. Kawagoe, T. Suemitsu, and M. Yokoyama, Light. Res. Technol. 34, 111 (2002).

${ }^{19}$ J. D. Rancourt and R. L. Martin, Jr., Proc. SPIE 678, 185-191 (1986).

${ }^{20}$ S. A. Spura, R. Fontana, L. J. Parascandola, W. E. Thouret, and L. Thorington, Thin Silver Film Coating for Increased Lamp Efficiency (Pergamon Press Canada Ltd., 1982).

${ }^{21}$ O. Ilic, P. Bermel, G. Chen, J. D. Joannopoulos, I. Celanovic, and M. Soljačić, Nat. Nanotechnol. 11, 320 (2016).

${ }^{22}$ General Electric. "GE light,” Datasheet 1 (2010).

${ }^{23}$ Y. S. Kim, S. Y. Lin, A. S. P. Chang, J. H. Lee, and K. M. Ho, J. Appl. Phys. 102, 063107 (2007).

${ }^{24}$ C. D. Tschetter and R. S. Bergman, "Incandescent lamp," U.S. patent, 4,535,269 (1985).

${ }^{25}$ W. K. Brinn, I. Berlec, J. M. Davenport, M. R. Vukcevich, and E. G. Fridrich, "Higher efficiency incandescent lighting units," U.S. patent, 4,547,704 (1985).

${ }^{26}$ L. E. Hoegler and L. W. Otto, "High efficiency tubular heat lamps," U.S. patent, 4,588,923 (1986)

${ }^{27}$ T. G. Parham, S. Desu, and C. D. Tschetter, "Tantala-silica interference filters and lamps using same," U.S. patent 4,949,005 (1990).
${ }^{28}$ F. J. Studer and D. A. Cusano, J. Opt. Soc. Am. 43, 522 (1953).

${ }^{29}$ R. P. Fontana, I. S. Goldstein, L. Thorington, and R. P. Howson, CIE J. 6, 11 (1987).

${ }^{30}$ R. L. Martin, Jr. and J. D. Rancourt, U.S. patent 4,663,557 A (1982).

${ }^{31}$ S. A. Spura, R. Fontana, L. J. Parascandola, W. E. Thouret, and L. Thorington, "Thin Silver Film Coating for Increased Lamp Efficiency," in Proc. 5th Intl. Precious Metals Institute Conf. (Precious Metals 1981) (Elsevier, 1982), pp. 259-264.

${ }^{32}$ B. E. Yoldas and T. O'Keefe, Appl. Opt. 23, 3638 (1984).

${ }^{33}$ J. Cho, J. H. Park, J. K. Kim, and E. F. Schubert, Laser Photonics Rev. 11, 1600147 (2017).

${ }^{34}$ V. Rinnerbauer, A. Lenert, D. M. Bierman, Y. X. Yeng, W. R. Chan, R. D. Geil, J. J. Senkevich, J. D. Joannopoulos, E. N. Wang, M. Soljačić, and I. Celanovic, Adv. Energy Mater. 4, 1400334 (2014).

${ }^{35}$ V. Stelmakh, V. Rinnerbauer, R. D. Geil, P. R. Aimone, J. J. Senkevich, J. D. Joannopoulos, M. Soljačić, and I. Celanovic, Appl. Phys. Lett. 103, 123903 (2013).

${ }^{36}$ V. Rinnerbauer, Y. X. Yeng, W. R. Chan, J. J. Senkevich, J. D. Joannopoulos, M. Soljačić, and I. Celanovic, Opt. Express 21, 11482 (2013).

${ }^{37}$ M. Aldrich, Dynamic Solid State Lighting (Massachusetts Institute of Technology, 2010).

${ }^{38}$ V. Rinnerbauer, E. Lausecker, F. Schäffler, P. Reininger, G. Strasser, R. D. Geil, J. D. Joannopoulos, M. Soljačić, and I. Celanovic, Optica 2, 743 (2015).

${ }^{39}$ See http://jdj.mit.edu/ ilic/public/css/reclight/recycling_light_additional_ materials.pdf for O. Ilic, "Additional Materials for "Tailoring high-temperature radiation and the resurrection of the incandescent source"' (last accessed May 20, 2017). 